\title{
A NOVEL IN-FLIGHT SPACE BATTERY HEALTH ASSESSMENT SYSTEM Brandon Buergler $^{(1)}$, François Bausier ${ }^{(1)}$
}

\author{
(1)ESA-ESTEC, Keplerlaan 1, 2200 AG Noordwijk, NL,Email: brandon.buergler@esa.int, francois.bausier@esa.int
}

\begin{abstract}
The assessment of an in-flight battery health status is of great interest. However, it is very difficult to perform with sufficient accuracy because the battery is constantly operated and no full discharge during a space mission can be done. Currently, a method for performing a health assessment is via the correlation of battery capacity with internal resistance, which can be more easily measured. Another method is to fit the on board battery voltage using the telemetry of current and temperature as inputs by advanced modelling techniques and to derive the ageing parameters of the battery. This is done by optimising the parameters so that the fitted voltage curve is as close as possible to the actual measured voltage telemetry curve. Then an assessment of the battery capacity and energy can be done by simulating a battery discharge at the desired conditions with the required voltage limits. However, these two methods often require extensive battery life tests on ground to estimate the ageing behaviour for a particular mission.

In this paper a novel health assessment system is proposed by which a more straight forward and accurate assessment of the battery health in terms of capacity and energy can be performed. The proposed system consists to use one or some battery string(s), which can be safely disconnected via the PCDU (Power Conditioning and Distribution Unit) from the battery for a dedicated capacity measurement while the main part of the battery continues to operate normally. In this paper, the viability of the proposed system is demonstrated and the implications on the battery and the power system are discussed.
\end{abstract}

\section{INTRODUCTION}

It is difficult to assess the health status of a spacecraft battery in operation. Due to design, operational and safety constraints, it is essentially impossible to fully discharge the battery in order to accurately measure the currently available capacity. The battery state of health in terms of capacity can be crucial when a mission is to be extended beyond the initially planned duration. In contrast, it is easier to assess the internal resistance of the battery. Every time an increasing current step occurs on the battery bus, the voltage across the battery terminals drops. The internal resistance of the battery can be calculated from this voltage drop and the difference of current before and after the step. Due to limited telemetry sampling rate in the range of one measurement every few seconds, the data has to be statistically evaluated. Only current steps with a certain threshold can be included in the evaluation. A prediction of the capacity fade of a spacecraft battery has then to be based on the relationship between the internal resistance and remaining capacity, which is probably a complex one. The basis for this approach is the availability of experimental data for the cells in similar conditions to the ones encountered in the spacecraft of which the remaining battery capacity is to be determined. This calls for the conduction of long duration life tests on single cells of representative batteries. In practice there is always a difference between the experimental test conditions and the real conditions of a satellite in operation. So the predicted capacity will almost certainly deviate from the real one. The subject is currently under discussion among the space power community worldwide and an adequate solution to this issue would be of great interest. Currently an ISO standard is being prepared on this topic (ISO/TR 20891 Space systems - Space batteries Guidelines for easing in-flight health assessment). The methods that are currently being used are mostly based on fitting the telemetry data of the in-flight battery by adapting the ageing parameters of the battery models. The derived values for capacity fade and resistance increase are then validated towards other telemetry data before an assessment of capacity and energy are done. Examples of this approach are given in references [1-4]. However, in practice it is difficult to achieve an accuracy better than $10-20 \%$ with these methods. If it were possible to assess the battery capacity with improved accuracy, it would be possible to better:

- know the battery health status in order to optimise the spacecraft operations, in particular in case of mission extension beyond the initially planned duration

- keep the spacecraft battery at a sufficiently high state of charge for nominal operations and recovery while mitigating the battery capacity fading

- get in-flight data about battery capacity fading in order to improve knowledge and optimise future space power systems

- investigate the cause of potential power system degradation or failure cases

\section{SYSTEM DESCRIPTION}

A new system for accurate battery health assessment is proposed here, in which the battery capacity is directly deduced from on board measurements. A scheme of the 
proposed system is shown in Figure 1. Within the battery, one or more "measurement" strings are connected separately via dedicated terminals leading to the PCDU or another electronic unit. In the following the solution is described assuming one measurement string, keeping in mind that it could be similarly implemented with two or more strings in parallel. This measurement string can be disconnected from the battery main part via the PCDU (via a switch) when the battery is fully charged (e.g. just before an eclipse or with a dedicated charge circuit). Then it can be fully discharged to determine the remaining capacity of the measurement string. A procedure for determining the internal resistance of the string(s) can also be implemented as well as a state of charge (SOC) determination from the measurement of the disconnected string electromotive force (EMF) at zero current. The advantage of this approach is that the satellite can remain in the nominal state but with one battery string less. No risky satellite manoeuvres nor solar array depointing nor battery deep discharge need to be done.

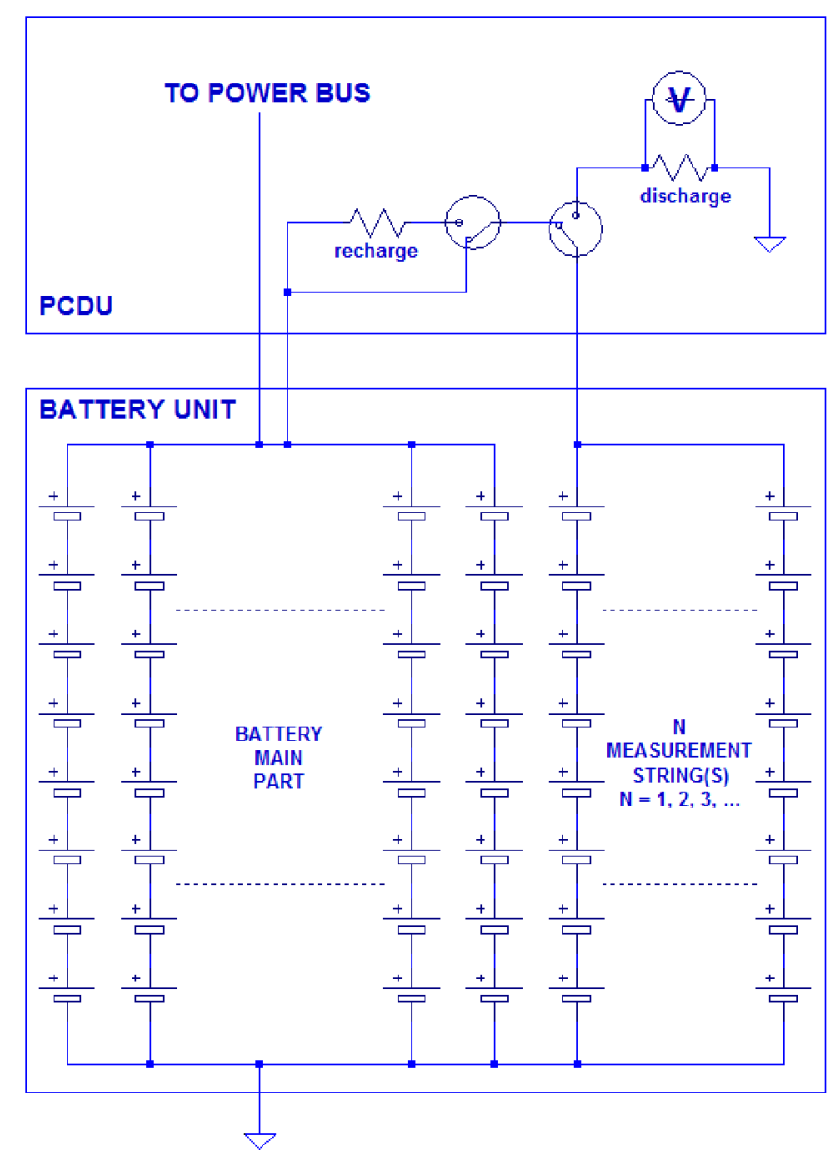

Figure 1 - Proposed battery and PCDU configuration for determining the capacity of an in-flight battery with one or more measurement string(s) connected via the PCDU to the main battery bus.
After recharging the measurement string to the bus voltage (e.g. via a resistor), the switch can be closed again and the string is reconnected to the other battery strings. This approach requires only small changes to the PCDU and to the batteries with a limited cost and weight impact. The telemetry system will have to include the current and voltage values of the individual string. From these telemetry values the actual battery capacity can be calculated. The discharge can be simply done by a resistor (in which case the current can be deduced with accuracy from the voltage telemetry) or by a current source to achieve constant current discharge.

Note that Figure 1 features two switches in order to cope with the accidental connection of the measurement string to the battery main part while the voltages are not equalized, which could stress the string. Some precautions or protection may also be foreseen to mitigate the risk to overdischarge cells in the measurement string.

Physically, the measurement string(s) could be located among the other strings at any chosen location in the battery in order to be exposed to representative environmental conditions.

\section{ASSESSMENT BY SIMULATION}

Some simulations have been done using the Power Energy Platform Simulation PEPS developed at ESTEC [5], in particular to check the influence of the harness and switch resistance on the cell current and voltage of the measurement string.

Figure 2 shows a circuit where one measurement string would be connected inside the PCDU after the battery harness during normal operation of the battery (i.e. no health check being done).

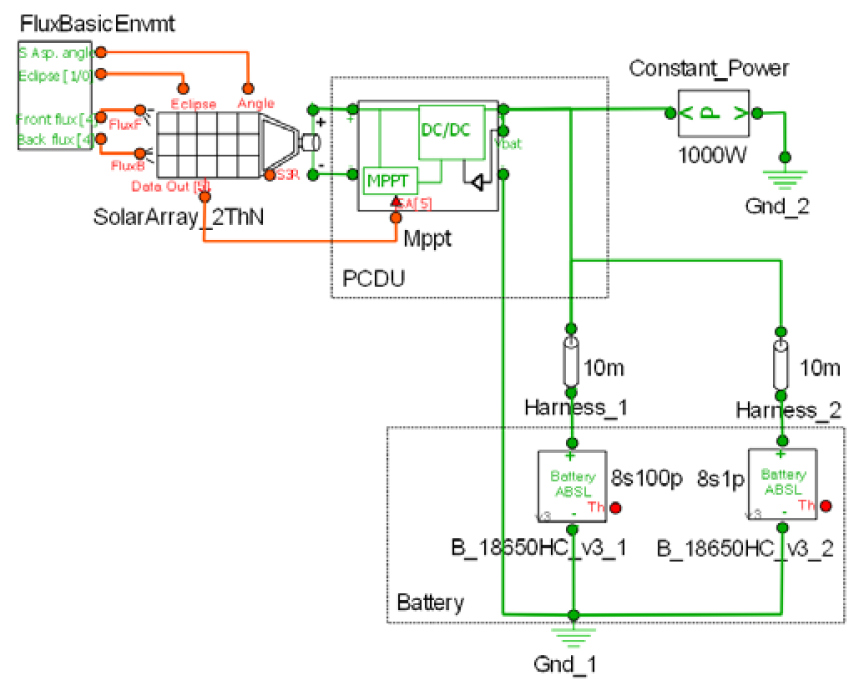

Figure 2 - Simulation diagram (measurement string connected after battery harness; no discharge circuit represented in this diagram). 
In this case the current and voltage of the string are not representative of the main battery strings (Figures 3 and 4). This is due to the difference of equivalent series resistance of the main battery strings ( $\mathrm{R}$ harness_main $\times$ number of strings in main battery) and of the measurement string $(\mathrm{R}$ _harness measurement string $\times$ 1). The single string is connected to the PCDU with a lower resistance as compared to a string in the main battery, which leads to a more severe cycling. An evaluation of battery capacity on the basis of such a system would lead to an overestimation of the battery capacity fading.

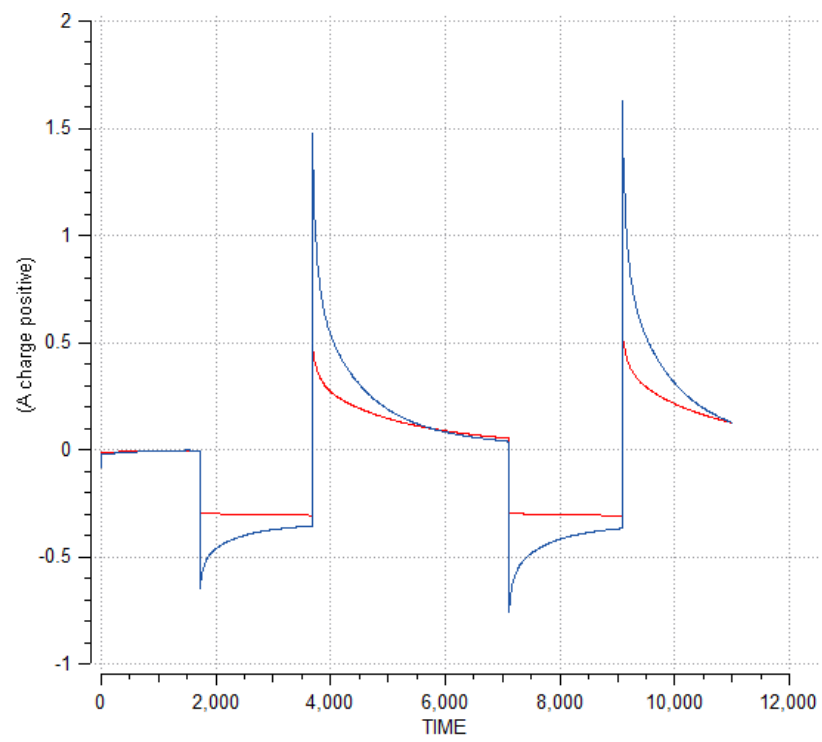

Figure 3 - Comparison of cell current during LEO eclipse cycles for diagram Fig 2 (red=battery main part, blue=measurement string). The measurement string is cycled with higher current than the strings in

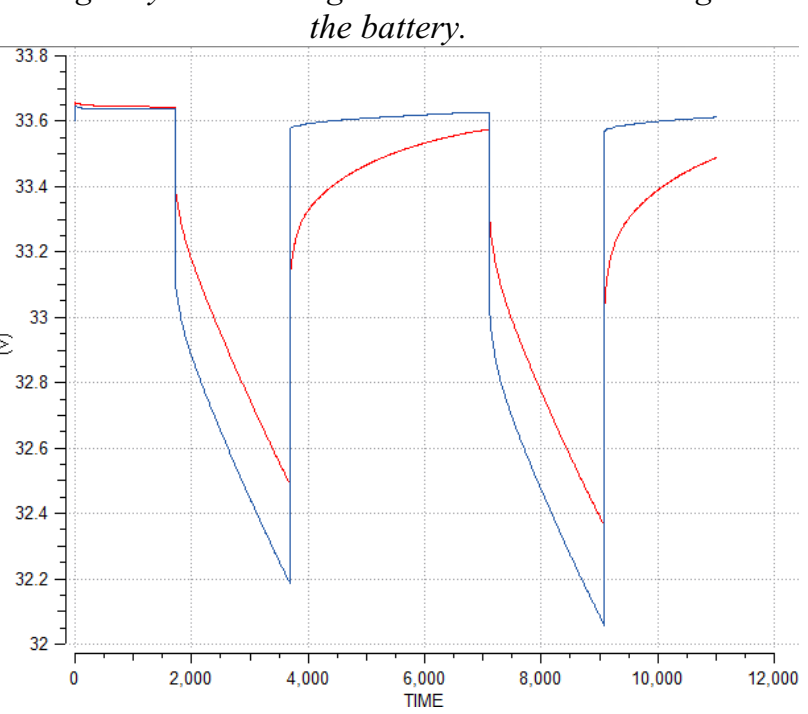

Figure 4 - Comparison of voltages during LEO eclipse cycles for diagram Fig 2 (red=battery main part, blue=measurement string). The measurement string is cycled with a larger voltage range than the strings in the battery.
A way to solve this issue would be to adjust $\mathrm{R}$ harness measurement_string equal to $\mathrm{R}$ - harness main $\mathrm{x}$ number of strings in main battery. However, in practice this solution is difficult to achieve with good accuracy.

A solution to mitigate the influence of the main harness and connection is to connect the measurement string on the battery internal bus bar as shown in Figure 5 .

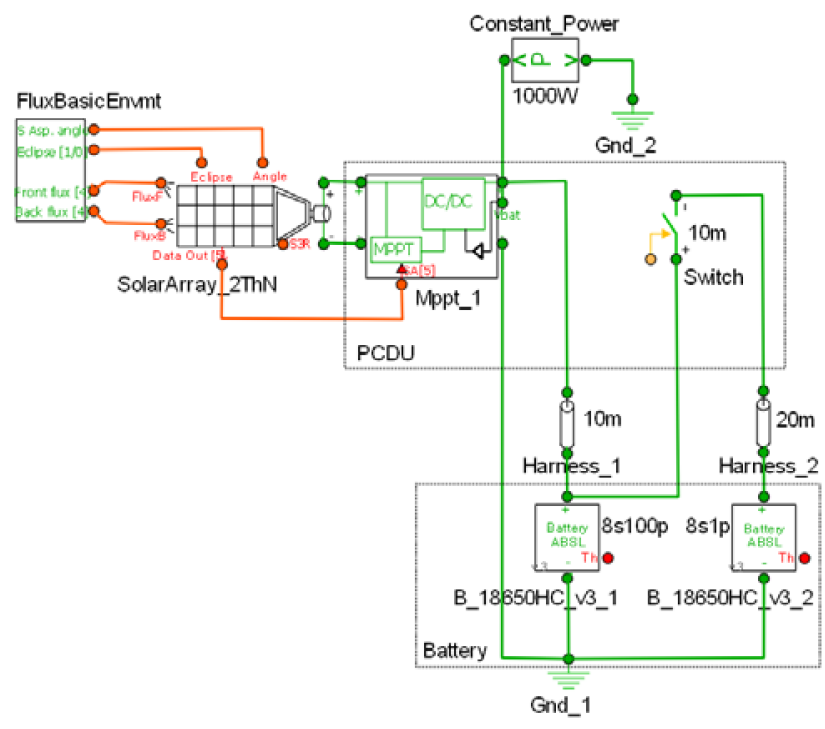

Figure 5 - Simulation diagram (measurement string connected before battery harness; no discharge circuit represented in this diagram).

The simulations performed on the basis of this improved configuration are shown in Figure 6 and Figure 7. In this case the currents and voltages of the cells in the measurements string are very similar to the ones in the battery main part.

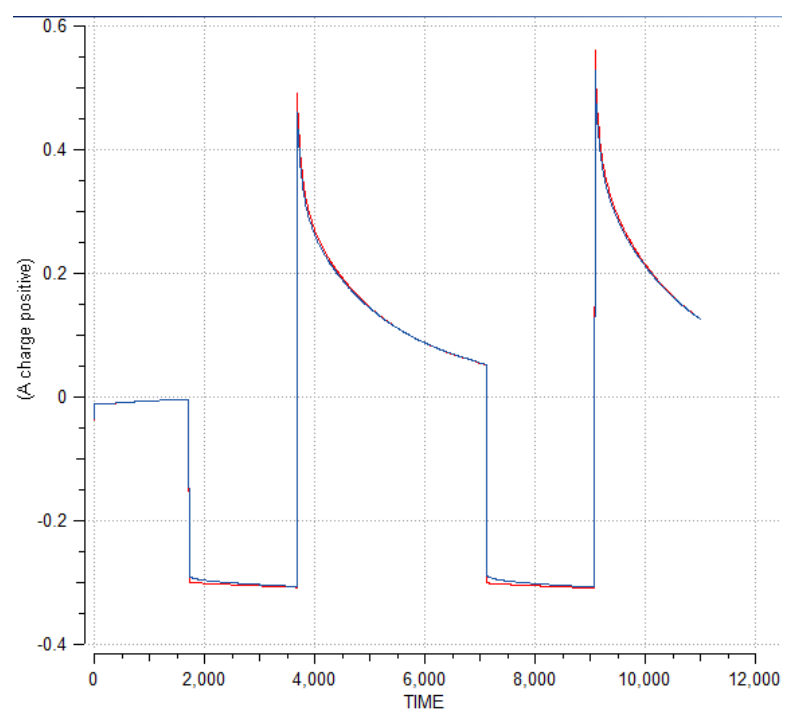

Figure 6-Comparison cell current during LEO eclipse cycles for diagram Fig 5 (red=battery main part, blue $=$ measurement string). 


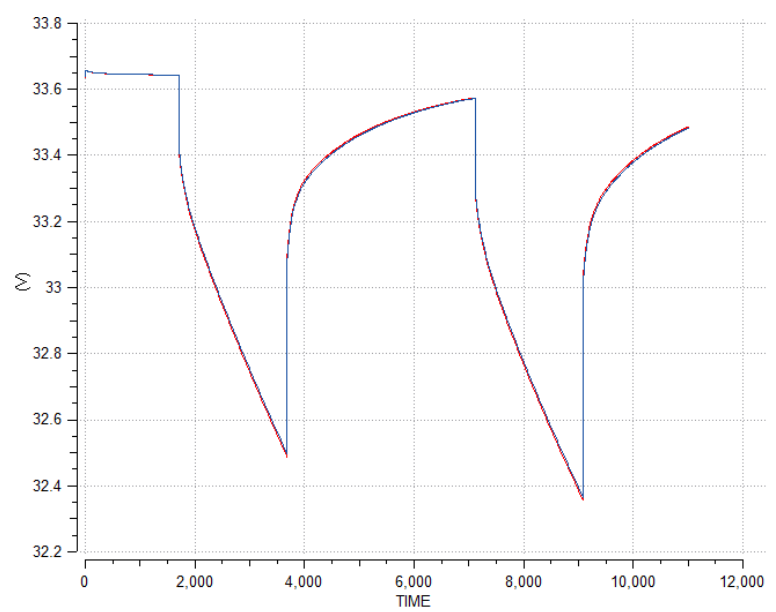

Figure 7 - Comparison of voltages during LEO eclipse cycles for diagram Fig 5 (red=battery main part, blue=measurement string)

The total connection resistance of the measurement string has to be as low as possible to achieve an acceptable match between this string and the strings in the main battery (same current and same voltage during cycling). The possibility to practically achieve a low enough resistance (much lower than the string resistance) and the influence of all parasitic resistances (including the switch resistances) have to be considered to obtain the desired accuracy. An additional resistance of $3 \%$ (TBC) for the measurement string as compared to the main battery strings seems to be a reasonable aim. A way to minimise the effect of this resistance is to connect several measurement strings in parallel inside the battery instead of only one. Finally the complete system including the discharge circuit has been simulated (Figure 8).

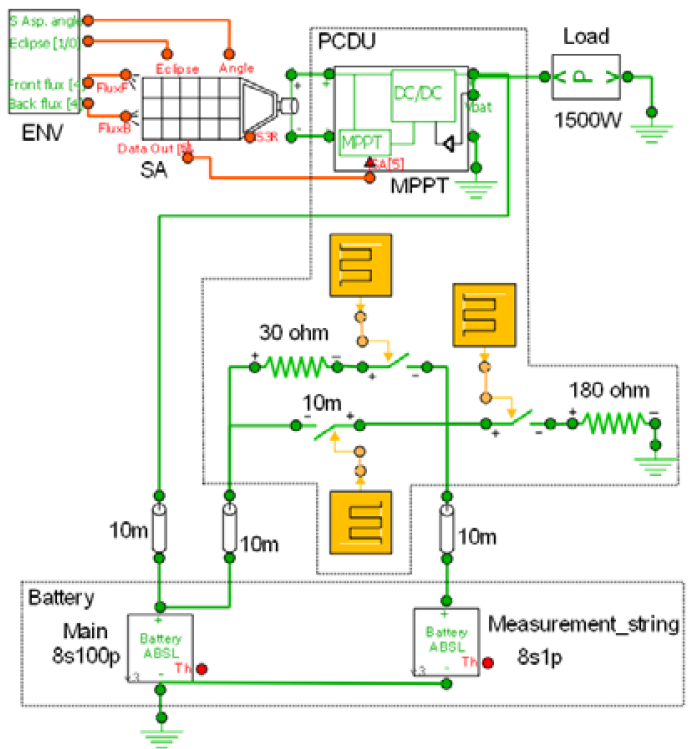

Figure 8 - Simulation diagram of complete system with one measurement string (battery capacity fading of $30 \%)$.
In this simulation diagram $3 \%$ additional harness resistance for the measurement string have been taken into account. The results are shown in Figures 9 to 11 .

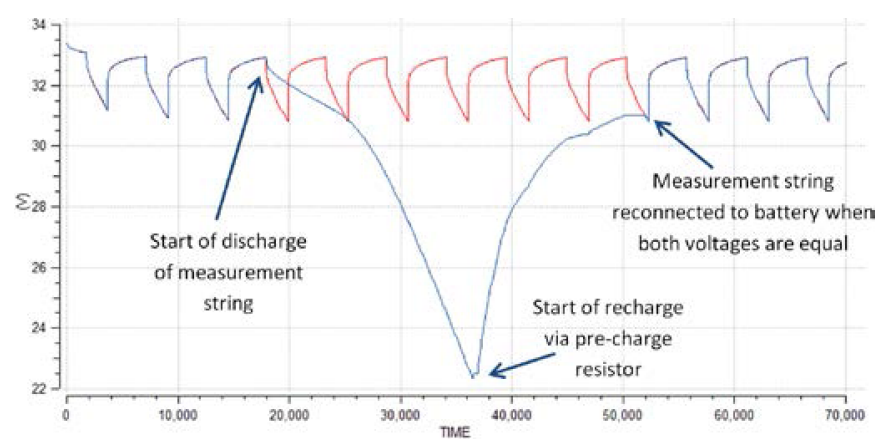

Figure 9-Battery voltage vs. measurement string voltage during LEO eclipse cycles.

Figure 9 shows that after some cycles the measurement string is disconnected from the main battery after having been fully charged (about $33 \mathrm{~V}$ battery voltage or $4.1 \mathrm{~V} /$ cell). The measurement string is then connected to the $180 \mathrm{ohm}$ resistor and is discharged at a current of about $\mathrm{C} / 10(150 \mathrm{~mA})$ down to the minimum voltage. The maximum power dissipated in this resistor is around $6 \mathrm{~W}$ at $33 \mathrm{~V}$. Ideally the discharge could be done down to $20 \mathrm{~V}(2.5 \mathrm{~V} /$ cell $)$ plus some safety margin. However, due to simulation model constraints it has been stopped at $22.5 \mathrm{~V}(2.8 \mathrm{~V} /$ cell $)$ in this simulation.
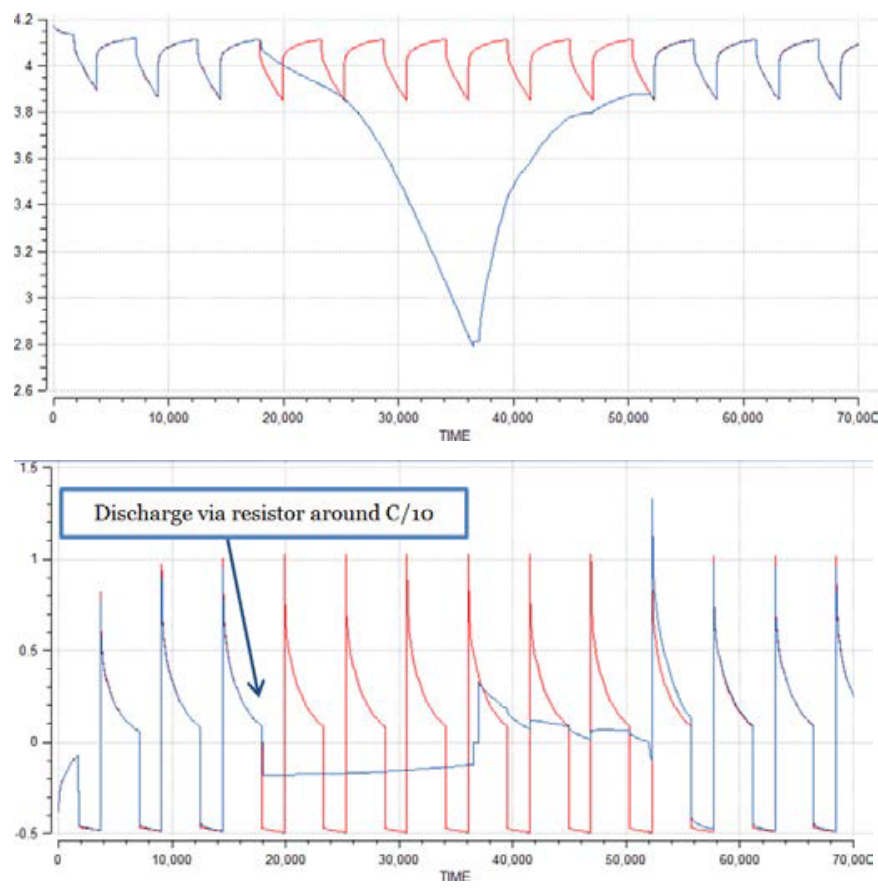

Figure 10-Cell voltage (top, [V]) and cell current (bottom, [A]) (red=battery main part, blue $=$ measurement string).

Then the measurement string is disconnected from the $180 \mathrm{ohm}$ resistor and connected to the pre-charge 
resistor $(30 \mathrm{ohm})$ until it reaches again the same voltage as the main battery. At this time it is reconnected to the main battery.

Based on the measurement string voltage and current telemetries it is possible to determine the remaining capacity (and internal resistance) of the measurement string and therefore of the main battery: in this case $70 \%$ of the BOL (Beginning of Life) capacity. Considering a telemetry accuracy better than $1 \%$ EOL (End of Life) e.g. based on high accuracy $0.1 \%$ resistors, an accuracy of the capacity/energy measurement better than a few percent can be expected. Figure 10 shows that the measurement string voltage and current satisfactorily mimic the main battery string voltage and current both before and after the full discharge/charge cycle of the measurement string. Only one or two eclipse cycles are necessary to recover the perfect matching.

In addition the SoC (State of Charge) is shown in Figure 11. Both measurement string cells and main battery cells behave the same way before and after the discharge/charge cycle.

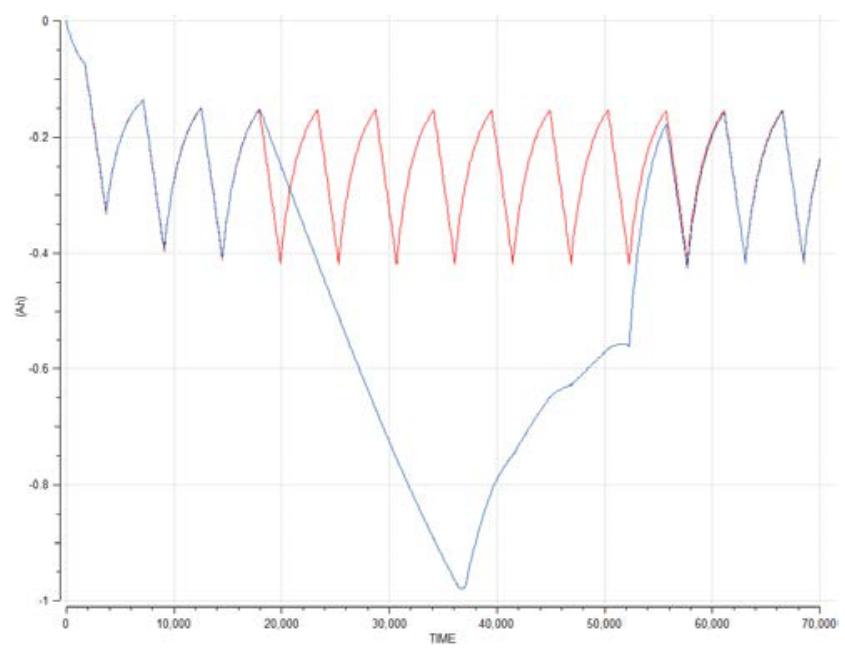

Figure 11 - Cell state of charge in Ah extracted from the cells (red=battery main part, blue=measurement string).

\section{CRITICAL ANALYSIS}

The last system configuration shown in Figure 8 is promising. An advantage of the system is that it is possible to fully discharge a representative part of the battery for obtaining the overall battery capacity. Capacity assessments on the basis of partial discharges only, have uncertainties concerning the full capacity. Open circuit voltage (OCV) measurements (i.e. battery voltage measurements at zero current) could also be done if needed. Some capacity determination methods are based on OCV measurements, which are not available on satellite batteries without the proposed system. However the following points are worth to be noted to optimise the system:
- The level of degradation of the measurement string due to the full discharge tests has to be considered. Obviously such full discharge test should not be performed too often; otherwise the measurement string would degrade more rapidly than the main battery ones (especially at BOL). For example a frequency of once a year would not degrade the measurement string significantly.

- In the measurement string there are already several cells and the discharged capacity of this string will already account for cells with higher and lower than average capacities. Thus, the measurement of one string will represent an average cell in the battery, however, without information about the variability from cell to cell. Having several measurement strings instead of one would allow for an even more representative capacity figure that could account for the locally different environment in a larger battery system including such with more than one module.

- The optimal number of measurement strings in parallel has to be determined on a case by case basis, also taking into account the series resistance achieved by the connection circuit via the PCDU, the level of fidelity desired and the cost.

- The number of switches needed in the connection/discharge circuit (via PCDU) may vary depending on the desired robustness against faulty battery connection and/or faulty discharge, which could potentially stress the measurement string(s) during AIT activities or in flight. Two switches in series for the battery connection and two switches in series for the discharge would offer a better robustness against a single failure or a wrong command.

- The way the switches are commanded may also vary according to the desired robustness. Two level commands (e.g. arm and fire) would offer a better robustness against a single failure or a wrong command.

- In case it is not convenient to fully charge the battery before the measurement, a partial discharge can also be performed. The remaining energy starting from the actual end of charge voltage during the mission (e.g. at $80 \%$ SOC) to the given battery end of discharge voltage seems to be a suitable reference energy for assessing the state of health as well.

- This new system was initially based on ABSL18650HC cell in sp (series-parallel) configuration where a large number of strings are in parallel. Nevertheless the same principle seems valid for other batteries for example based on Saft VES16 or MPS (sp configuration) or even larger Saft cells (ps configuration) with balancing electronics. However, dedicated balancing electronics need to be foreseen for the measurement string(s). Note that this string contribute to the nominal battery performance, except during the string full discharge 
test where some operational measures can be taken if necessary (in case the measurement string represents a significant part of the whole battery).

- The detailed impacts on the overall power system in terms of FMECA (Failure Modes, Effects and Criticality Analysis), hardware needed, passivation, representativeness of the selected string, battery bus variation during recharge of separated string and so forth need yet to be determined in detail. Finally tests will be needed to verify the expected performances.

\section{IMPACT ON BATTERY AND PCDU}

For the battery itself and the PCDU the following adaptions would have to be made:

\section{Battery:}

Internally the battery design could be the same but one (or more) string(s) would have to be taken from the main battery internal bus on the positive side.

In order to connect the measurement string to the PCDU and back to the main battery positive bus, two extra pins (or more depending on the resistance of the connection) are needed. These pins could be provided on the already existing battery power connector or it could be placed on a dedicated connector.

PCDU:

As shown on Figure 1 the PCDU would have to include:

- Switches (e.g. relays) including commands to connect the measurement string to the main battery, to the discharge circuit or to the pre-charge resistor (with redundancy for operational/safety reasons).

- A discharge circuit: the simplest is a resistor (with voltage telemetry allowing to measure both voltage and current with high accuracy) but a current source could also be implemented to achieve constant current discharge.

- A pre-charge resistor (to recharge the measurement string to the main battery voltage before to connect to it).

- Two extra pins (or more) to connect the measurement string.

\section{CONCLUSIONS}

A new system for determining with accuracy the health status of an in-flight space battery has been described with an expected accuracy of better than a few percent. No modelling or simulations, which induce inaccuracies are needed for the capacity determination. Expensive long duration life tests on cell or battery level can also be avoided to some extent. The measurement string current during the capacity assessment can be controlled independently of the satellite operations.

There is currently an increasing interest in the space power community to get such an accurate battery health assessment solution.

Preliminary assessments and analyses of this new system have been conducted and the outcome is promising.

\section{REFERENCES}

[1] D. Genc, C. Thwaite, "Proba-1 and Mars Express: An ABSL Lithium-Ion Legacy", European Space Power Conference, (2011).

[2] D. Curzon, C. Thwaite, R. Buckle, "Lithium-Ion Battery Performance on board geostationary and lunar orbiter NASA missions - SDO and LRO", European Space Power Conference, (2011).

[3] Y. Borthomieu, D. Gambini, "Saft Li-Ion VES140S Battery Flight Experience Return on TAS Space Bus Platforms", 10th European Space Power Conference, (2014).

[4] Final report TRP activity "Battery capacity estimation based on modelling and advanced data processing techniques" , ESA Contract Nr. 4000114068. [5] A. Fernandez, G. D’Accolti, B. Buergler, B. Garcia, "PEPS. A Tool for Power System Simulation", 10th European Space Power Conference, ESA, Noordwijkerhout, The Netherlands, 2014 